\title{
Predictors of early postoperative epileptic seizures after awake surgery in supratentorial diffuse gliomas
}

\author{
Marc Zanello, MD, MSc, ${ }^{1-3}$ Alexandre Roux, MD, MSc, ${ }^{1-3}$ Gilles Zah-Bi, MD, MSc, ${ }^{1-3}$ \\ Bénédicte Trancart, MSc, ${ }^{1-3}$ Eduardo Parraga, MD, ${ }^{1-3}$ Myriam Edjlali, MD, PhD, ${ }^{2-4}$ \\ Arnault Tauziede-Espariat, MD, , 2,3,5 Xavier Sauvageon, MD, ,3,6 Tarek Sharshar, MD, PhD, 2,3,6,7 \\ Catherine Oppenheim, MD, PhD, ${ }^{2-4}$ Pascale Varlet, MD, PhD, ${ }^{2,3,5}$ Edouard Dezamis, MD, MSc, ${ }^{1-3}$ \\ and Johan Pallud, MD, PhD ${ }^{1-3}$
}

\begin{abstract}
1Department of Neurosurgery, Sainte-Anne Hospital, Paris; ${ }^{2}$ Paris Descartes University, Sorbonne Paris Cité; ${ }^{3}$ nserm U1266, IMA-Brain, Centre de Psychiatrie et Neurosciences, Paris; Departments of ${ }^{4}$ Neuroradiology, ${ }^{5}$ Neuropathology, and

${ }^{6}$ Neuro-Anaesthesia and Neuro-Intensive Care, Sainte-Anne Hospital, Paris; and ${ }^{7}$ Laboratory of Experimental Neuropathology,

Pasteur Institute 28, Paris, France
\end{abstract}

\begin{abstract}
OBJECTIVE Functional-based resection under awake conditions had been associated with a nonnegligible rate of intraoperative and postoperative epileptic seizures. The authors assessed the incidence of intraoperative and early postoperative epileptic seizures after functional-based resection under awake conditions.

METHODS The authors prospectively assessed intraoperative and postoperative seizures (within 1 month) together with clinical, imaging, surgical, histopathological, and follow-up data for 202 consecutive diffuse glioma adult patients who underwent a functional-based resection under awake conditions.
\end{abstract}

\begin{abstract}
RESULTS Intraoperative seizures occurred in 3.5\% of patients during cortical stimulation; all resolved without any procedure being discontinued. No predictor of intraoperative seizures was identified. Early postoperative seizures occurred in $7.9 \%$ of patients at a mean of $5.1 \pm 2.9$ days. They increased the duration of hospital stay $(p=0.018)$, did not impact the 6-month (median 95 vs 100, $p=0.740$ ) or the 2-year (median 100 vs 100, $p=0.243$ ) postoperative Karnofsky Performance Status score and did not impact the 6 -month (100\% vs $91.4 \%, p=0.252)$ or the 2 -year $(91.7$ vs $89.4 \%, p=0.857)$ postoperative seizure control. The time to treatment of at least 3 months (adjusted OR [aOR] 4.76 [95\% Cl 1.38-16.36], p $=0.013$ ), frontal lobe involvement (aOR 4.88 [95\% Cl 1.25-19.03], $p=0.023$ ), current intensity for intraoperative mapping of at least $3 \mathrm{~mA}$ (aOR 4.11 [95\% Cl 1.17-14.49], $p=0.028$ ), and supratotal resection (aOR 6.24 [95\% Cl 1.43-27.29], $p=$ $0.015)$ were independently associated with early postoperative seizures.
\end{abstract}

CONCLUSIONS Functional-based resection under awake conditions can be safely performed with a very low rate of intraoperative and early postoperative seizures and good 6-month and 2-year postoperative seizure outcomes. Intraoperatively, the use of the lowest current threshold producing reproducible responses is mandatory to reduce seizure occurrence intraoperatively and in the early postoperative period.

https://thejns.org/doi/abs/10.3171/2020.1.JNS192774

KEYWORDS awake surgery; epileptic seizure; glioma; intraoperative; postoperative; oncology; epilepsy

$\mathrm{R}$ ESECTION is the first treatment for WHO diffuse gliomas no matter the grade of malignancy. It increases survival and preserves, or improves, healthrelated quality of life. These benefits should be carefully weighed against the operative risks. Surgery of diffuse gliomas, which infiltrate cortical and subcortical connectivity, is at high risk of neurological and cognitive deficits postoperatively. This has practical implications since survival of glioma patients is correlated with the quality of the resection and performance status of the patient. ${ }^{17}$ To this end, the use of intraoperative functional brain mapping using direct electrical stimulation under awake conditions is mandatory. ${ }^{6,24}$ The widespread use of this technique, which is the gold standard for diffuse glioma resection, can be limited by perioperative seizures, which have been reported as a regular complication impacting the surgical procedure and postoperative course. ${ }^{4,18,19,27,29,33}$ However, little is known regarding the predictors of intraoperative seizures and early postoperative seizures, and previous reports are biased by varying intraoperative brain mapping 
techniques that are known to impact the occurrence and observation of epileptic seizures. ${ }^{29}$

Here, we prospectively assessed the occurrence of intraoperative and postoperative seizures during the 1st operative month in adult patients who underwent resection of a diffuse glioma with intraoperative functional brain mapping using direct electrical stimulation under awake conditions. ${ }^{24}$

\section{Methods}

\section{Study Design and Setting}

This prospective, observational study was conducted at a tertiary referral neurosurgical center between January 2010 and December 2018. The local institutional review board approved the study protocol. Informed written consent was obtained from all patients prior to enrollment. This paper was written according to the Strengthening the Reporting of Observational Studies in Epidemiology (STROBE) checklist. ${ }^{32}$

\section{Participants}

Inclusion criteria at the time of surgery were 1) age $\geq$ 18 years; 2) tumor in a supratentorial location; 3 ) resection with intraoperative functional brain mapping using direct electrical stimulation under awake conditions; and 4) diffuse astrocytic or oligodendroglial tumors classified or reevaluated according to the 2016 update of the WHO classification..$^{12}$ We prospectively included 202 adult patients, corresponding to $100 \%$ of all eligible patients according to the selection criteria.

\section{Variables and Data Sources}

Patient-, surgery-, and tumor-related characteristics included sex, age, time to treatment (from first symptom to surgery), clinical sign(s) (increased intracranial pressure, focal neurological deficit, cognitive impairment, and epileptic seizure), number of seizures before surgery, seizure control, antiepileptic drug therapy, Karnofsky Performance Status (KPS) score, glioma location, contrast enhancement on MRI, ${ }^{22}$ hyperperfusion on MRI, glioma volume, duration of surgery, intraoperative seizure, current intensity for intraoperative mapping, subtype and grade of malignancy according to the 2016 update of the WHO classification (histopathological reassessment for all cases diagnosed prior to September 2016 ${ }^{12}$ ), IDHI/2 mutation status, oligodendroglioma $I D H$-mutant and $1 \mathrm{p} / 19 \mathrm{q}-$ codeleted, extent of resection, early postoperative seizure, duration of hospital stay, mode of discharge, 6-month and 2-year postoperative seizure control, and 6-month and 2-year KPS scores.

As previously detailed, ${ }^{22}$ the glioma volume was quantified by segmentation of signal abnormalities on the FLAIR sequence for grade II and III gliomas and of signal abnormalities on the postcontrast T1-weighted sequence for grade IV gliomas. The volume of residual glioma was quantified on early postoperative MRI (within 48 hours). For grade II and III gliomas, the extent of resection was defined as "total" when no signal abnormality was present, "supratotal" when a margin of parenchyma was removed around the FLAIR abnormality, and "subtotal" when the signal abnormality was less than $10 \mathrm{~cm}^{3} \cdot 3,36$ For grade IV gliomas, the extent of resection was defined as "total" when $100 \%$ of the contrast enhancement was removed, "supratotal" when $100 \%$ of the contrast enhancement plus an additional removal of peripheral FLAIR signal abnormality was removed, ${ }^{13}$ and "subtotal" when more than $90 \%$ of the contrast enhancement was removed. All other cases were considered as partial resection.

Epileptic seizures were diagnosed on a clinical basis only. Epileptic seizure types were classified according to the International League Against Epilepsy. ${ }^{9}$ Epileptic seizure control was defined as a patient completely free of any epileptic seizure with or without antiepileptic drug therapy. ${ }^{34}$

\section{Surgical Procedure}

All patients were operated on by the same two senior neurosurgeons (J.P. and E.D.). Intraoperative functional mapping was performed by cortical and subcortical direct bipolar electrical stimulation under awake conditions, using our in-house "asleep-awake-asleep" protocol, which has previously been detailed. ${ }^{17,24}$ On the day of the surgery, no anxiolytic or sedative medication was given, and antiepileptic drugs were administered as usual on an individual basis. The air temperature was controlled, and a forced-air heating blanket maintained the patient's temperature $>36^{\circ} \mathrm{C}$. Total intravenous general anesthesia with a laryngeal mask airway was performed using propofol (target concentration of $6 \mathrm{mg} / \mathrm{mL}$ ) and remifentanil (target concentration of $6 \mathrm{ng} / \mathrm{mL}$ ). Drug concentrations of these agents were gradually decreased to $3 \mathrm{mg} / \mathrm{mL}$ and $4 \mathrm{ng} / \mathrm{mL}$, respectively, just before anesthesia discontinuation. Pressure-controlled ventilation was used, with pressure maintained at $<15 \mathrm{~cm} \mathrm{H}_{2} \mathrm{O}$ to ensure normocapnia. Intravenous antiemetics, analgesics, antibiotic prophylaxis, steroids, and an intravenous loading dose of levetiracetam $(500 \mathrm{mg})$ were administered at induction. The scalp was infiltrated at the incision area, reflection area, and head holder pins with a local anesthetic ( $20 \mathrm{~mL}$ lidocaine $2 \%$ with epinephrine).

For functional mapping, we used a bipolar electrode with a 5-mm space between tips and a biphasic current (pulse frequency $60 \mathrm{~Hz}$, pulse phase duration $1 \mathrm{msec}$; Osiris NeuroStimulator, Inomed). No electrophysiological monitoring was performed, and all events were recorded on a clinical basis. The exposed brain was tested, and all sites were restimulated 3 times for confirmation. The current intensity was determined individually with a progressive increase of the amplitude by $0.5-\mathrm{mA}$ increments until a functional response was elicited (baseline $1 \mathrm{~mA}$ ). The cortical mapping began by stimulating the sensory/motor area to identify the lowest current threshold producing reproducible positive responses. The same current intensity served for further functional mapping.

To control for neurological and cognitive function, intraoperative tasks were performed (motor network, somatosensory network, language, calculation, memory or even visuospatial processing, semantic cognition, and social cognition) depending on glioma location and according to practical guidelines, ${ }^{11,23}$ and the types of disturbances were classified by a senior speech therapist. Each site inducing a functional impairment was marked with 
a sterile numbered tag. Then, the glioma was removed by alternating resection and stimulation for subcortical functional mapping in an awake patient performing the required tasks all along the glioma resection. Resections were stopped when eloquent subcortical structures were encountered within the surgical cavity with no security margin of brain tissue left around the functional connectivity or until the patient felt too tired to work efficiently.

The tips to control for intraoperative seizures that have been implemented in our surgical technique are detailed in Table 1. If an intraoperative epileptic seizure was clinically observed, stimulations were stopped immediately, no systemic anticonvulsant was administered, and the brain was irrigated with cold $\left(4^{\circ} \mathrm{C}-8^{\circ} \mathrm{C}\right)$ normal saline solution $(0.9 \% \mathrm{NaCl})$ until the seizures stopped. Resection was resumed as soon as the patient was able to perform cognitive tasks.

Postoperatively, patients received steroids if deemed necessary by the neurosurgeon; no systematic prophylaxis with antiepileptic drugs was administered, and the antiepileptic drug therapy was continued at the same dose as that given preoperatively.

\section{Statistical Analysis}

Descriptive statistics are given as the mean \pm standard deviation for continuous variables and as a percentage for categorical variables. To determine factors impacting intraoperative and early postoperative seizures, univariate analyses were carried out using the chi-square test or Fisher's exact test for comparing categorical variables and the unpaired t-test or Mann-Whitney rank-sum test for continuous variables, as appropriate. Variables associated at the $\mathrm{p}<0.2$ level in unadjusted analysis were then entered into multivariate logistic regression models. A p value < 0.05 was considered significant. Analyses were performed using JMP software version 14.1.0 (SAS Institute Inc.).

\section{Results \\ Study Population}

Complete records were available for the 202 patients under study. Their main characteristics are detailed in Table 2. At surgery, 144 patients $(71.3 \%)$ presented with epileptic seizures, of which $82.3 \%$ were controlled under antiepileptic drug therapy (1 drug in $84.5 \%$ and 2 drugs in $15.5 \%$; levetiracetam in $83.3 \%$ of cases). Of the 58 patients without epileptic seizures, $20(34.5 \%)$ were on antiepileptic drug therapy (levetiracetam in $52.9 \%$ of cases) administered prophylactically by the referring neuro-oncologist before arrival at our institution. During surgery, all patients were cooperative, there were no challenges that precluded the functional-based resection to be performed, and no patient required termination of the procedure. Intraoperatively, positive functional mapping was applied in all patients at a mean stimulation current intensity of $3.1 \pm$ $0.8 \mathrm{~mA}$ (range 1.0-6.0 mA).

\section{Intraoperative Epileptic Seizures}

Epileptic seizures occurred intraoperatively in 7 patients $(3.5 \%$; 6 focal onset without impaired awareness and 1 focal to bilateral tonic-clonic; semiology was coherent
TABLE 1. Surgical tips to improve intraoperative seizure management

\begin{tabular}{|c|}
\hline Surgical Tip \\
\hline $\begin{array}{l}\text { No change in the antiepileptic drug therapy administered preopera- } \\
\text { tively }\end{array}$ \\
\hline $\begin{array}{l}\text { Additional loading dose of levetiracetam during the induction phase } \\
\text { of the surgery }\end{array}$ \\
\hline $\begin{array}{l}\text { Positive mapping with exposure of the sensory/motor areas and } \\
\text { ventral premotor cortex }\end{array}$ \\
\hline $\begin{array}{l}\text { Calibration of the current intensity as the lowest current threshold } \\
\text { producing reproducible responses }\end{array}$ \\
\hline $\begin{array}{l}\text { Definition of an upper security limit of current intensity ( } 8 \mathrm{~mA} \text {, not } \\
\text { reached in the present study) }\end{array}$ \\
\hline $\begin{array}{l}\text { Lowest and same current intensity maintained during cortical and } \\
\text { subcortical mapping, in all locations, in all functional tasks }\end{array}$ \\
\hline Duration of direct electrical stimulation never exceeding 4 seconds \\
\hline Same brain area never stimulated twice in succession \\
\hline $\begin{array}{l}\text { Three sham stimulations following a direct electrical stimulation } \\
\text { inducing a clinical response }\end{array}$ \\
\hline $\begin{array}{l}\text { In cases of intraoperative seizure, no administration of systemic } \\
\text { anticonvulsant, just cold irrigation }\end{array}$ \\
\hline
\end{tabular}

with the site of stimulation in all cases) during cortical stimulation and before resection. All resolved after cold irrigation. No surgical procedure was discontinued, and all patients were able to pursue intraoperative tasks after their seizure. In multivariate analysis, no parameter was independently associated with intraoperative seizures.

\section{Early Postoperative Seizures}

Early postoperative epileptic seizures occurred in 16 patients $(7.9 \% ; 7$ focal to bilateral tonic-clonic, 5 focal onset without impaired awareness, and 4 focal onset with impaired awareness) at a mean of $5.1 \pm 2.9$ postoperative days (range 1-11 days) during the hospital stay in 15 cases and following discharge in 1 case. None of them had an intraoperative epileptic seizure. One patient had a status epilepticus, leading to a 12-day-long stay in the intensive care unit with sedation. The main characteristics of the patients with early postoperative epileptic seizures are detailed in Table 3.

The occurrence of early postoperative seizures significantly increased the duration of hospital stay (median 11.5 vs 9.0 days, $\mathrm{p}=0.018$ ) but did not significantly impact the rate of discharge to home after surgery $(68.8 \%$ vs $84.4 \%$, $\mathrm{p}=0.137$ ). At 6 months postoperatively, postoperative seizures did not significantly impact the KPS score (median 95 vs $100, \mathrm{p}=0.740)$ or the seizure control rate $(100 \%$ vs $91.4 \%, p=0.252)$. At 2 years postoperatively, they did not significantly impact the KPS score (median 100 vs 100, p $=0.243)$ or the seizure control rate $(91.7 \%$ vs $89.4 \%, \mathrm{p}=$ $0.857)$. Of note, in the subgroup of 16 patients with early postoperative seizures, the only patient with uncontrolled seizures at 2 years postoperatively had undergone partial removal of a left frontocentral WHO grade II oligodendroglioma, which required adjuvant chemotherapy. 
TABLE 2. Characteristics of the 202 patients under study

\begin{tabular}{|c|c|}
\hline & Value \\
\hline \multicolumn{2}{|l|}{ Sex } \\
\hline Male & $114(56.4)$ \\
\hline Female & $88(43.6)$ \\
\hline Mean age \pm SD (range), yrs & $45.2 \pm 14.7(14-74)$ \\
\hline Mean BMI \pm SD (range) & $24.2 \pm 4.2(17.0-39.5)$ \\
\hline \multicolumn{2}{|l|}{ Time btwn diagnosis \& op, mos } \\
\hline Mean \pm SD (range) & $33.3 \pm 12.9(0-224)$ \\
\hline$<3$ & $116(57.4)$ \\
\hline$\geq 3$ & $86(45.6)$ \\
\hline \multicolumn{2}{|l|}{ Increased intracranial pressure } \\
\hline No & $185(91.6)$ \\
\hline Yes & $17(8.4)$ \\
\hline \multicolumn{2}{|l|}{ Focal neurological deficit } \\
\hline No & $152(75.2)$ \\
\hline Yes & $50(24.8)$ \\
\hline \multicolumn{2}{|l|}{ Cognitive deficit } \\
\hline No & $166(82.2)$ \\
\hline Yes & $36(17.8)$ \\
\hline \multicolumn{2}{|l|}{ Epileptic seizure } \\
\hline No & $58(28.7)$ \\
\hline Yes & $144(71.3)$ \\
\hline \multicolumn{2}{|l|}{ Seizure control* } \\
\hline No & $25(17.4)$ \\
\hline Yes & $119(82.6)$ \\
\hline \multicolumn{2}{|l|}{ No. of AEDs } \\
\hline None & $38(18.8)$ \\
\hline 1 & $133(65.8)$ \\
\hline$\geq 2$ & $31(15.4)$ \\
\hline \multicolumn{2}{|l|}{ KPS score } \\
\hline Mean \pm SD (range) & $92.8 \pm 11.9(50-100)$ \\
\hline$\geq 70$ & $197(97.7)$ \\
\hline$<70$ & $5(2.3)$ \\
\hline \multicolumn{2}{|l|}{ Main lobar location } \\
\hline Frontal & $100(49.5)$ \\
\hline Temporal & $45(22.3)$ \\
\hline Parietal & $32(15.8)$ \\
\hline Insular & $23(11.4)$ \\
\hline Other & $2(1.0)$ \\
\hline \multicolumn{2}{|l|}{ Frontal lobe involvement } \\
\hline No & $72(35.6)$ \\
\hline Yes & $130(64.4)$ \\
\hline \multicolumn{2}{|l|}{ Insular lobe involvement } \\
\hline No & $147(72.8)$ \\
\hline Yes & $55(27.2)$ \\
\hline \multicolumn{2}{|l|}{ Contrast enhancement on MRI } \\
\hline No & $98(48.3)$ \\
\hline Faint \& patchy & $33(16.4)$ \\
\hline Nodular-like & $44(21.9)$ \\
\hline Ring-like w/ central necrosis & $27(13.4)$ \\
\hline
\end{tabular}

CONTINUED IN NEXT COLUMN »
» CONTINUED FROM PREVIOUS COLUMN

TABLE 2. Characteristics of the 202 patients under study

\begin{tabular}{lc}
\hline \multicolumn{2}{l}{ Value } \\
\hline Hyperperfusion on MRI & $94(46.5)$ \\
\hline No & $108(53.5)$ \\
\hline Yes & $66.6 \pm 72.6(1-227.2)$ \\
\hline Mean glioma vol \pm SD (range), $\mathrm{cm}^{3}$ & $267 \pm 76(140-523)$ \\
\hline Mean overall op duration \pm SD (range), mins & \\
\hline Mean duration of awake phase w/ functional & $99 \pm 32(40-245)$ \\
mapping \pm SD (range), mins & \\
\hline Intraop seizure & $7(3.5)$ \\
\hline No & \\
\hline Yes & $3.1 \pm 0.8(1.0-6.0)$ \\
\hline Current intensity for functional mapping, mA & $142(70.3)$ \\
\hline Mean \pm SD (range) & $60(29.7)$ \\
\hline$\leq 3$ &
\end{tabular}

Histopathological grade \& subtype (WHO 2016)
II, IDH-mut oligodendroglioma
$29(14.4)$
II, IDH-mut astrocytoma
$38(18.8)$
II, IDH-wt astrocytoma
5 (2.5)
III, IDH-mut oligodendroglioma
$41(20.3)$
III, IDH-mut astrocytoma
$38(18.8)$
III, IDH-wt astrocytoma
$3(1.5)$
IV, IDH-mut glioblastoma
$13(6.4)$
IV, IDH-wt glioblastoma
$35(17.3)$

$I D H$-mutated \& 1p19q co-deleted oligodendroglioma

\begin{tabular}{cr} 
No & $132(65.3)$ \\
Yes & $70(34.7)$ \\
\hline IDH1/2 mutation &
\end{tabular}

No $43(21.3)$

Yes

$159(78.7)$

Extent of resection

Partial $52(25.7)$

Subtotal $\quad 53(26.2)$

Total $\quad 73(36.2)$

Supratotal 24 (11.9)

Early postop seizure

No $186(92.1)$

Yes $16(7.9)$

Mean LOS \pm SD (range), days $\quad 10.5 \pm 5.7$ (4-51)

Discharge

Home 168 (83.2)

Institution 34 (16.8)

6-mo postop seizure control

No $16(7.9)$

Yes $186(92.1)$

CONTINUED ON PAGE 687 » 
》 CONTINUED FROM PAGE 686

TABLE 2. Characteristics of the 202 patients under study

\begin{tabular}{cc}
\hline \multicolumn{2}{c}{ Value } \\
\hline 6-mo postop KPS score & \\
\hline Mean \pm SD (range) & $91.5 \pm 14.0(5-100)$ \\
\hline$\geq 70$ & $190(94.1)$ \\
\hline$<70$ & $12(5.9)$ \\
\hline 2 -yr postop seizure controlt & $16(9.7)$ \\
\hline No & $149(90.3)$ \\
\hline Yes & \\
\hline 2 -yr postop KPS score $\dagger$ & $86.0 \pm 22.4(40-100)$ \\
\hline Mean \pm SD (range) & $140(84.8)$ \\
\hline$\geq 70$ & $25(15.2)$ \\
\hline$<70$ &
\end{tabular}

AED = antiepileptic drug; LOS = length of stay; mut = mutant; $w t=$ wild type.

Values represent the number of patients (\%) unless stated otherwise.

* In patients with seizures at the time of surgery.

$\dagger$ In patients with an available 2-year follow-up.

\section{Predictors of Early Postoperative Seizures}

In multivariate analysis, the time to treatment of longer than 3 months (adjusted OR [aOR] 4.76 [95\% CI 1.38$16.36], \mathrm{p}=0.013$ ), frontal lobe involvement (aOR $4.88[95 \%$ CI 1.25-19.03], $\mathrm{p}=0.023$ ), current intensity for intraoperative mapping of higher than $3 \mathrm{~mA}$ (aOR 4.11 [95\% CI 1.17-14.49], $\mathrm{p}=0.028)$, and a supratotal resection beyond FLAIR abnormalities (aOR 6.24 [95\% CI 1.43-27.29], $\mathrm{p}=$ 0.015 ) were independently associated with early postoperative seizures in the overall population (Table 4) and in the subgroup of $I D H$-mutated diffuse gliomas (Table 5). We observed no significant impact of age $(\mathrm{p}=0.953)$, presence of epileptic seizure $(p=0.434)$, seizure control $(p=0.683)$, use of antiepileptic drug therapy $(p=0.212)$, number of antiepileptic drugs $(\mathrm{p}=0.283)$, insular lobe involvement ( $\mathrm{p}$ $=0.138)$, temporal lobe involvement $(p=0.104)$, duration of the awake phase of the surgery ( $p=0.987)$, occurrence of intraoperative seizures ( $p=0.330)$, or histopathological diagnosis $(\mathrm{p}=0.551)$. Of note, although 5 of the 38 patients $(13.2 \%)$ without antiepileptic drug therapy administered at the time of surgery and 11 of the 164 patients (6.7\%) with antiepileptic drug therapy administered at the time of surgery had early postoperative seizures, the use of antiepileptic drug therapy was not a significant predictor of early postoperative seizures $(\mathrm{p}=0.212)$.

\section{Discussion}

\section{Key Results}

In this prospective cohort study including 202 adult patients harboring a supratentorial diffuse glioma and undergoing a functional-based maximal resection using intraoperative direct bipolar electrical stimulation under awake conditions, we found the following. 1) Intraoperative epileptic seizures are an extremely rare event (3.5\%) using our protocol ${ }^{24}$ that resolve systematically after cold irrigation and do not impact the surgical procedure. 2) No predictors of intraoperative seizures are identified. 3) Early postoperative epileptic seizures are a rare event (7.9\%) that occur during the first 11 postoperative days and impact the duration of hospital stay. 4) Early postoperative epileptic seizures do not impact the postoperative KPS score or seizure control at 6 months or 2 years. 5) Time to treatment longer than 3 months, frontal lobe involvement, current intensity for intraoperative mapping higher than $3 \mathrm{~mA}$, and supratotal resection beyond FLAIR abnormalities are independent predictors of early postoperative seizures.

\section{Interpretation}

The low rate of intraoperative seizures that did not impact the functional brain mapping suggests the feasibility and safety of the surgical procedure, even in patients with epileptic seizures at surgery. The present results recall those from the Montpellier experience, which reported a $3.4 \%$ rate of intraoperative seizures with no discontinuation of the surgery in 361 diffuse glioma patients operated on using the same surgical methodology under awake conditions. ${ }^{4}$ The low rate of intraoperative seizures we have observed in these two French studies challenges previous publications, which reported intraoperative seizures as a main complication of intraoperative brain mapping with an incidence of up to $54 \%$, leading to discontinuation of the procedure in up to $18 \% .4,8,18,19,25,27,29,33$ Here, intraoperative seizures were identified on a clinical basis only without the use of electrophysiological recordings, which may explain the various rates in comparison with previous reports in which intraoperative seizures were identified using electrophysiological recordings. It is known that the occurrence of intraoperative seizures during functional brain mapping is linked with technical and practical aspects of direct electrical stimulation applied to the brain and, particularly, the current intensity. ${ }^{31}$ As a consequence, and in agreement with methodological guidelines, ${ }^{4,6,23,31,36}$ we implemented our surgical technique to reduce intraoperative seizure occurrence, as detailed in Methods. ${ }^{24,25}$ Contrary to previous reports, ${ }^{8,29}$ the implementation of positive mapping (performed in all cases) in our intraoperative functional mapping strategy did not induce a high rate of intraoperative seizures. Similar to the Montpellier experience, we experienced a $3.5 \%$ incidence of intraoperative seizures without intraoperative electrophysiological monitoring, including electrocorticographic recordings, ${ }^{4}$ suggesting that clinically relevant afterdischarges can be controlled by a strict methodology of direct brain electrical stimulation, including the initial calibration of the lowest current intensity. This recalls the work of Spena et al., who reported a significant link between the mastery of the intraoperative functional brain mapping technique and the risk of intraoperative seizures. ${ }^{29}$ The presence of epileptic seizures, the presence of uncontrolled seizures at surgery, and the number of antiepileptic drug therapies have previously been reported as associated ${ }^{18,29}$ or not $^{4,22}$ with a higher incidence of intraoperative seizures. The lack of clear evidence in this specific question has been highlighted in a recent comprehensive review of stimulation-related intraoperative seizures during awake surgery. ${ }^{25} \mathrm{Here}$, we failed to identify predictors of intraoperative seizures, possibly linked with the low number of observed events. 


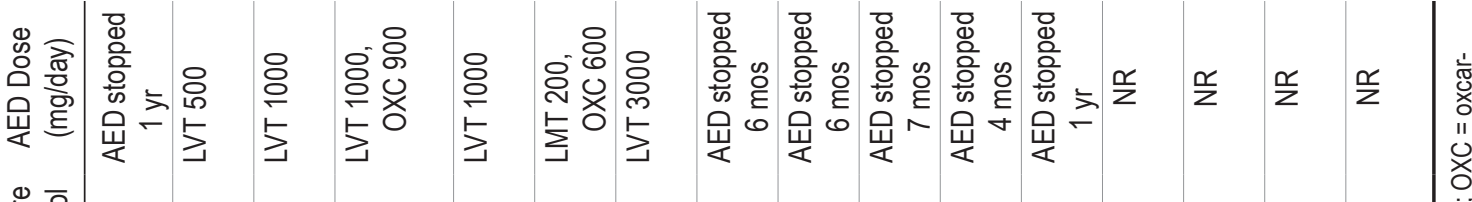

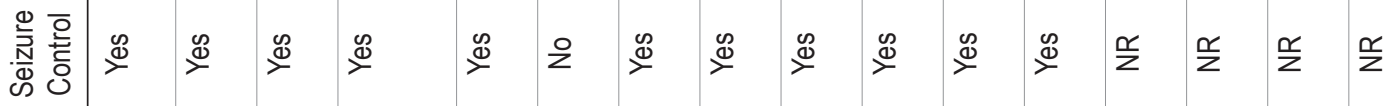

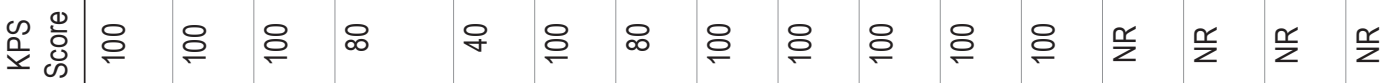

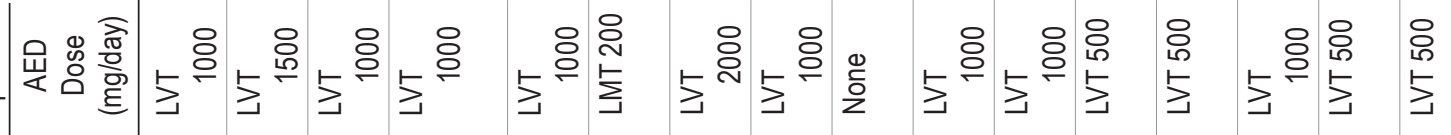

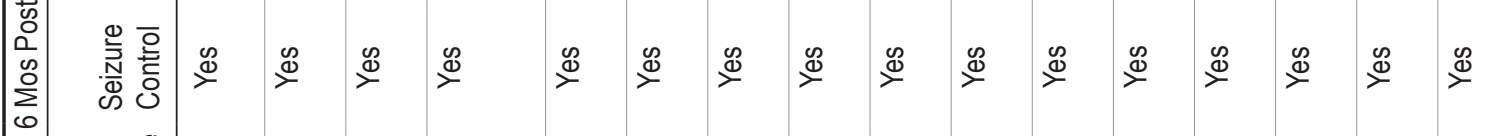

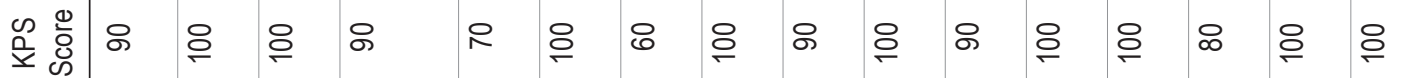

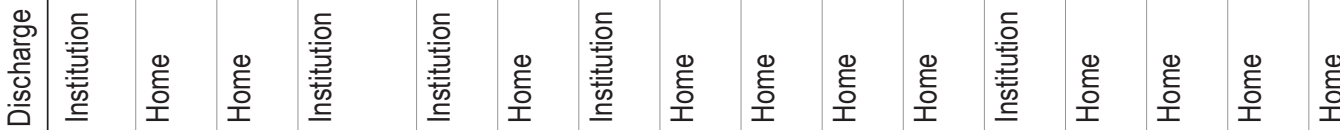

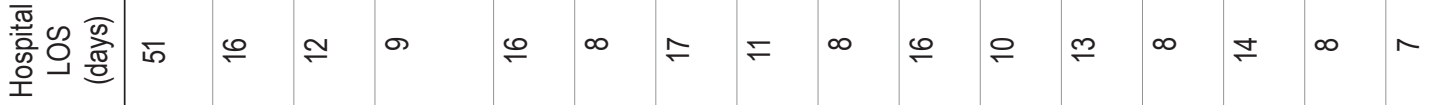

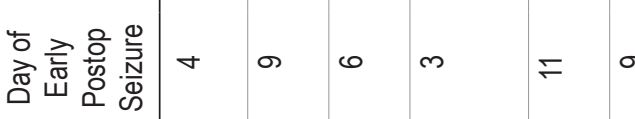

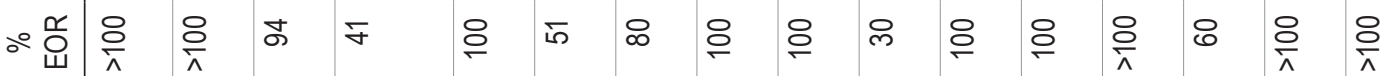

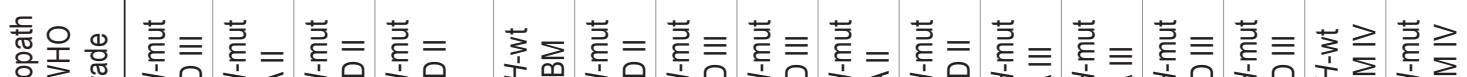

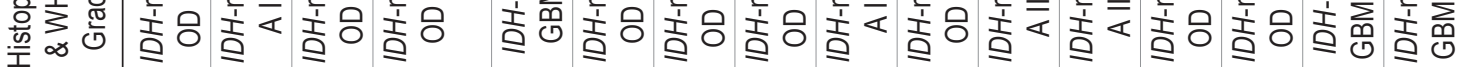

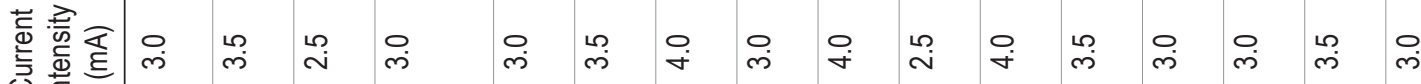

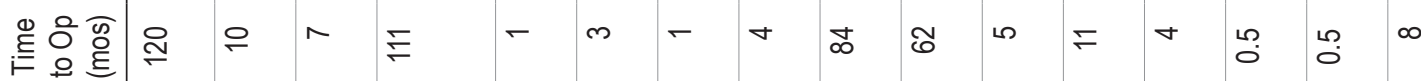

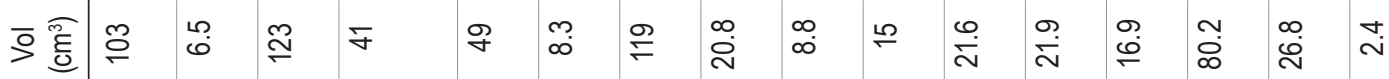

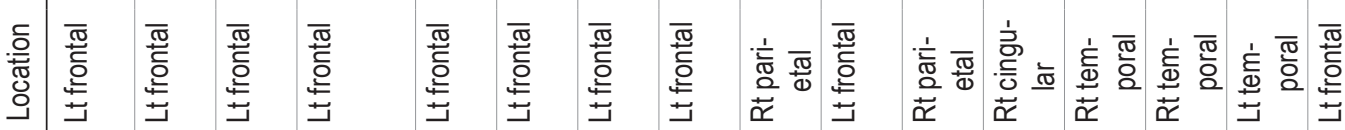

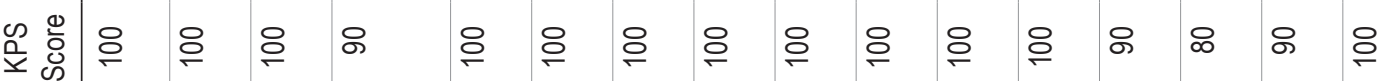

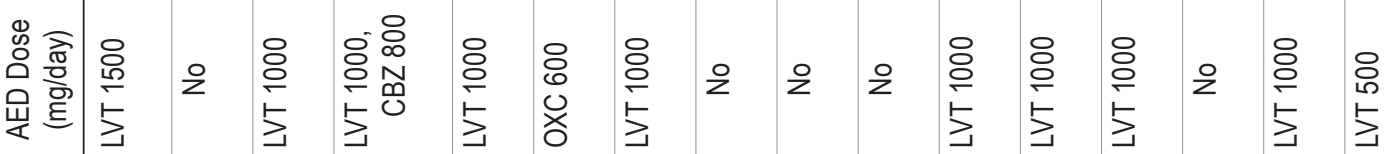

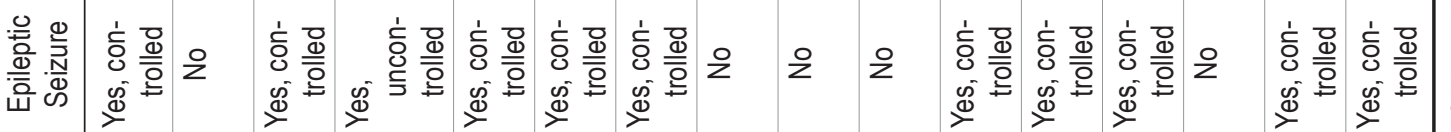

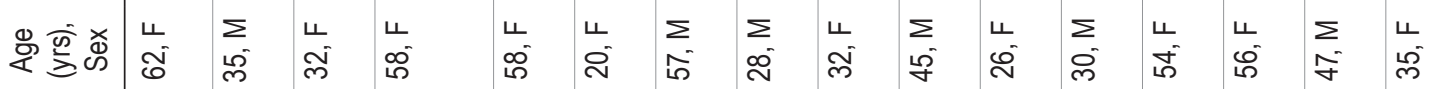
Di 
TABLE 4. Risk factors of early postoperative epileptic seizures in 202 patients after glioma functional-based resection using direct electrical stimulation under awake condition

\begin{tabular}{cccc}
\hline \multicolumn{1}{c}{ Parameter } & aOR & $95 \% \mathrm{Cl}$ & $\mathrm{p} \mathrm{Value}$ \\
\hline Time to treatment, mos & \multicolumn{3}{c}{} \\
\hline$<3$ & Ref & & \\
\hline$\geq 3$ & 4.76 & $1.38-16.36$ & 0.013 \\
\hline Frontal lobe location & & & \\
\hline No & Ref & & \\
\hline Yes & 4.88 & $1.25-19.03$ & 0.023 \\
\hline Current intensity for intraop mapping, mA & & & \\
\hline$<3$ & Ref & & \\
\hline$\geq 3$ & 4.11 & $1.17-14.49$ & 0.028 \\
\hline
\end{tabular}

Supratotal resection beyond FLAIR-

defined abnormalities

\begin{tabular}{llll} 
No & Ref & & \\
Yes & 6.24 & $1.43-27.29$ & 0.015 \\
\hline
\end{tabular}

Multivariate backward stepwise logistic regression model. Also entered in the model but not significant: sex, temporal lobe location, insular lobe location, glioma volume on FLAIR sequence, oligodendroglioma subtype, presence of epileptic seizure at surgery, controlled seizures at surgery, use of antiepileptic drugs at surgery, number of antiepileptic drugs at surgery, duration of the awake phase, and occurrence of intraoperative seizures.

Little is known regarding early postoperative seizures following resection under awake conditions. We report a $7.9 \%$ rate of early postoperative epileptic seizures in 202 patients, which is within the range of previous reports, as detailed in Table 6. ${ }^{3,5,10,14,16,20,23,28,35,37}$ Little evidence exists regarding the clinical consequences of early postoperative seizures. We observed that early postoperative seizures had short-term clinical impacts similar to those that were previously reported: increased duration of hospital stay, and reduced rate of discharge to home after surgery. ${ }^{5,33}$ However, early postoperative epileptic seizures had no significant long-term clinical impact; the 6-month and 2-year postoperative KPS score and seizure control rate were not different from those without early postoperative seizures. This contrasts with a previous study that suggested early postoperative seizures were an independent risk factor for 6-month postoperative seizure recurrence. ${ }^{1,33}$ No previous study has addressed the identification of risk factors of early postoperative seizures in patients harboring a supratentorial diffuse glioma and operated on using intraoperative functional brain mapping under awake conditions. The identification of a time to treatment of longer than 3 months as an independent predictor of early postoperative seizures may be explained by two factors. First, the time required to develop epileptogenicity, which is mainly triggered by interactions between glioma cells and neocortex, ${ }^{29,30}$ and, second, by a longer time to treatment in patients harboring a lower-grade diffuse glioma, which are most prone to cause seizures. The identification of a frontal lobe involvement as a predictor of early postoperative seizures may be explained by the high epileptogenicity of premotor cortex and primary motor cortex ${ }^{26}$ which was previously described for intraoperative seizures. ${ }^{25}$ The
TABLE 5. Risk factors of early postoperative epileptic seizures after glioma functional-based resection using direct electrical stimulation under awake condition in the subgroup of IDH-mutated glioma $(\mathrm{n}=159)$

\begin{tabular}{cccc}
\hline \multicolumn{1}{c}{ Parameter } & aOR & $95 \% \mathrm{Cl}$ & $\mathrm{p}$ Value \\
\hline Time to treatment, mos & & & \\
\hline$<3$ & Ref & & \\
\hline$\geq 3$ & 5.13 & $1.22-21.62$ & 0.026 \\
\hline Frontal lobe location & & & \\
\hline No & Ref & & \\
\hline Yes & 6.85 & $1.41-33.19$ & 0.017 \\
\hline Current intensity for intraop mapping, mA & & & \\
\hline$<3$ & Ref & & \\
\hline$\geq 3$ & 5.34 & $1.31-21.73$ & 0.019 \\
\hline
\end{tabular}

Supratotal resection beyond FLAIR-

defined abnormalities

\begin{tabular}{llll} 
No & Ref & & \\
Yes & 5.05 & $1.18-21.66$ & 0.029 \\
\hline
\end{tabular}

Multivariate backward stepwise logistic regression model. Also entered in the model but not significant: sex, temporal lobe location, insular lobe location, glioma volume on FLAIR sequence, oligodendroglioma subtype, presence of epileptic seizure at surgery, controlled seizures at surgery, use of antiepileptic drugs at surgery, number of antiepileptic drugs at surgery, duration of the awake phase, and occurrence of intraoperative seizures.

identification of the current intensity of higher than $3 \mathrm{~mA}$ as an independent predictor of early postoperative seizures, which has previously been identified as a risk factor for intraoperative seizures,${ }^{34}$ is difficult to explain. We can only speculate on the induction of long-lasting electrophysiological changes induced by the high current intensity and on tumor-induced changes in the cortex requiring the current intensity to be increased to obtain reproducible responses. This result justifies the use of the lowest current threshold producing reproducible responses during intraoperative mapping to reduce the seizure occurrence perioperatively. The extent of resection, and particularly the supratotal resection beyond FLAIR abnormalities, is an independent predictor of early postoperative seizures, similar to findings in a previous report. ${ }^{33}$ This may be explained by the spatial organization of epileptogenic foci at the border between the glioma per se and the surrounding functional neocortex microscopically invaded by isolated glioma cells ${ }^{29,30}$ that are transiently enhanced by surgically related acute changes in the resection borders. Alternatively, this may be explained by the fact that supratotal resections are more often achieved in patients with incidentally discovered diffuse gliomas, ${ }^{15,17,28}$ in whom an antiepileptic drug prophylaxis is not usually administered. Of note, the observed link between resection and seizures in the early postoperative period contrasts with results at 6 months and 2 years postoperatively where the extent of resection is a significant predictor of seizure control. ${ }^{21,30}$ The early postoperative seizures we observed following supratotal resection did not compromise the good 6-month and 2-year postoperative functional outcomes (based on KPS score) and seizure outcomes and did not reduce its sur- 
TABLE 6. Studies reporting early postoperative seizures after glioma resection

\begin{tabular}{|c|c|c|c|c|c|c|}
\hline \multirow[b]{2}{*}{ Authors \& Year } & \multirow[b]{2}{*}{ Study Design } & \multirow[b]{2}{*}{ No. of Cases } & \multirow[b]{2}{*}{ Awake Surgery } & \multirow[b]{2}{*}{ Glioma } & \multicolumn{2}{|c|}{ Postop } \\
\hline & & & & & Seizure Rate & Follow-Up (days) \\
\hline Present series & Prospective & 202 & $100 \%$ & $100 \%$ & $7.9 \%$ & 30 \\
\hline Wang et al., 2019 & Retrospective & 41 & $100 \%$ & $100 \%$ & $34.1 \%$ & 7 \\
\hline Oushy et al., 2018 & Retrospective & 549 (no seizure history) & $11.6 \%$ (cortical stimulation) & NA & $4.6 \%$ & 30 \\
\hline Pallud \& Dezamis, 2017 & Prospective & 107 & $100 \%$ & $100 \%$ & $7.5 \%$ & 30 \\
\hline Dewan et al., 2017 & Retrospective & 342 & NA & $100 \%$ & $5.3 \%$ & 14 \\
\hline Lima \& Duffau, 2015 & Retrospective & 19 & $100 \%$ & $100 \%$ & $10.5 \%$ & 30 \\
\hline Mandonnet et al., 2015 & Retrospective & 24 & $100 \%$ & $100 \%$ & $12.5 \%$ & NA \\
\hline Skardelly et al., 2015 & Retrospective & 492 & NA & $41.3 \%$ & $7.4 \%$ & 7 \\
\hline Gokhale et al., 2013 & Retrospective & 165 & NA & $59.4 \%$ & $9.2 \%$ & 7 \\
\hline Wu et al., 2013 & Prospective & 123 & NA & $37.4 \%$ & $17.4 \%$ & 30 \\
\hline Zachenhofer et al., 2011 & Retrospective & 78 & NA & $52.6 \%$ & $2.5 \%$ & 7 \\
\hline
\end{tabular}

$\mathrm{NA}=$ not available

vival benefit. ${ }^{7,13,36}$ Interestingly, the preoperative epileptic seizure status and preoperative antiepileptic drug therapy did not impact the occurrence of early postoperative seizure. The nonsignificant lower incidence of early postoperative seizures in patients with antiepileptic drug therapy administered at the time of surgery recalls the previous literature. ${ }^{10,37}$ Our data failed to analyze the impact of antiepileptic drug therapy administered at the time of surgery in preventing early postoperative seizures. This important question requires further investigation in larger prospective and controlled studies.

Altogether, this suggests that early postoperative seizures corresponded to acute symptomatic postoperative seizures due to the resection and not to seizures due to the glioma itself since they did not significantly impact the long-term postoperative seizure control or the functional status of the patients. Here, the observed interval between the surgery and the early postoperative seizure ranged from 1 to 11 days, similar to a previous observation ranging from 0 to 13 days. ${ }^{5}$ These results may help refine the definition of acute symptomatic seizures according to the International League Against Epilepsy, which recently considered seizures as acute symptomatic ones if they occurred within the first 7 days following intracranial surgery. ${ }^{2}$

As a practical message, these predictors may help preoperatively identify patients who are at higher risk of early postoperative seizure. This, together with the observation of more frequent early postoperative seizures in patients who did not receive antiepileptic drug therapy at the time of surgery, allows discussing the introduction of a systematic antiepileptic drug prophylaxis or the increase in antiepileptic drug dosage during the perioperative period in these patient subgroups, as previously proposed for the perioperative management of incidentally discovered diffuse gliomas. ${ }^{14,15}$

\section{Limitations}

These findings should be interpreted with the full knowledge of the limitations arising from the diagnosis of intraoperative seizures and of early postoperative seizures on a clinical basis only and the number of observed events that limited the number of variables to be entered in multivariate models.

\section{Generalizability}

In previous studies, different brain tumor types (including meningioma and metastasis together with diffuse gliomas) and varying surgical techniques used (intraoperative brain mapping performed or not, asleep vs awake conditions) impacted the occurrence of perioperative epileptic seizures, rendering difficult the evaluation of the actual impact of direct electrical stimulation under awake conditions. This bias was controlled by selecting a homogeneous population of diffuse gliomas in adult patients who all underwent a functional-based resection using intraoperative direct bipolar electrical stimulations under awake conditions by the same two neurosurgeons using the same procedure. In addition, we reassessed all diffuse gliomas under study according to the 2016 update of the WHO classification. The standardized data collection and the absence of loss to follow-up allowed for a reliable evaluation of the effect of resection.

\section{Conclusions}

Maximal functional-based resection using intraoperative functional mapping under awake conditions can be safely and reproducibly performed with a very low rate of intraoperative and early postoperative seizures, and with excellent postoperative seizure outcomes, regardless of the epileptic seizure status at the time of surgery. Intraoperatively, it requires a rigorous surgical methodology to reduce the seizure occurrence intraoperatively and in the early postoperative period.

\section{Acknowledgments}

We gratefully acknowledge (in alphabetical order): the surgical neuro-oncology team of the Sainte-Anne Hospital, Paris, and particularly Aurore Gendre, Amel Khallouf, Odile Rigaux-Viodé, and Sylvie Sicot; the Department of Neurosurgery of the Sainte- 
Anne Hospital, Paris; the Department of Neuropathology of the Sainte-Anne Hospital, Paris; the Department of Neuroradiology of the Sainte-Anne Hospital, Paris; the Department of Neurophysiology of the Sainte-Anne Hospital, Paris, and particularly Martine Gavaret and Angela Marchi; the Department of Neuro-Anaesthesia and Neuro-Intensive Care of the SainteAnne Hospital, Paris, and particularly Abderrezak Akhrouf, Roland Benichou, Serge Biou, Hortense Dumontier, Aurélie Gruner, Aurélien Mazeraud, Alain Monpetit, Caroline Schimpf, Alain Sermet, and Gilles Thouvenot; and the Neuro-Oncology Unit of the Gustave Roussy University Hospital, Villejuif, France, and particularly Frédéric Dhermain and Sarah Dumont.

Marc Zanello, Alexandre Roux, and Johan Pallud are grateful to Hugues Duffau for his constructive reading of this manuscript.

This article is dedicated to the memory of William "mon p'tit Willi" Naessens who participated in the early years of awake surgery at Sainte-Anne Hospital.

\section{References}

1. Bech KT, Seyedi JF, Schulz M, Poulsen FR, Pedersen CB: The risk of developing seizures before and after primary brain surgery of low- and high-grade gliomas. Clin Neurol Neurosurg 169:185-191, 2018

2. Beghi E, Carpio A, Forsgren L, Hesdorffer DC, Malmgren K, Sander JW, et al: Recommendation for a definition of acute symptomatic seizure. Epilepsia 51:671-675, 2010

3. Berger MS, Deliganis AV, Dobbins J, Keles GE: The effect of extent of resection on recurrence in patients with low grade cerebral hemisphere gliomas. Cancer 74:1784-1791, 1994

4. Boetto J, Bertram L, Moulinié G, Herbet G, Moritz-Gasser S, Duffau H: Low rate of intraoperative seizures during awake craniotomy in a prospective cohort with 374 supratentorial brain lesions: electrocorticography is not mandatory. World Neurosurg 84:1838-1844, 2015

5. Dewan MC, White-Dzuro GA, Brinson PR, Zuckerman SL, Morone PJ, Thompson RC, et al: The influence of perioperative seizure prophylaxis on seizure rate and hospital quality metrics following glioma resection. Neurosurgery 80:563570, 2017

6. Duffau H: Diffuse low-grade glioma, oncological outcome and quality of life: a surgical perspective. Curr Opin Oncol 30:383-389, 2018

7. Duffau H: Long-term outcomes after supratotal resection of diffuse low-grade gliomas: a consecutive series with 11-year follow-up. Acta Neurochir (Wien) 158:51-58, 2016

8. Eseonu CI, Rincon-Torroella J, Lee YM, ReFaey K, Tripathi $\mathrm{P}$, Quinones-Hinojosa A: Intraoperative seizures in awake craniotomy for perirolandic glioma resections that undergo cortical mapping. J Neurol Surg A Cent Eur Neurosurg 79:239-246, 2018

9. Fisher RS, Cross JH, French JA, Higurashi N, Hirsch E, Jansen FE, et al: Operational classification of seizure types by the International League Against Epilepsy: Position Paper of the ILAE Commission for Classification and Terminology. Epilepsia 58:522-530, 2017

10. Gokhale S, Khan SA, Agrawal A, Friedman AH, McDonagh DL: Levetiracetam seizure prophylaxis in craniotomy patients at high risk for postoperative seizures. Asian J Neurosurg 8:169-173, 2013

11. Herbet G, Rigaux-Viodé O, Moritz-Gasser S: Peri- and intraoperative cognitive and language assessment for surgical resection in brain eloquent structures. Neurochirurgie 63:135-141, 2017

12. International Agency for Research on Cancer: WHO Classification of Tumours of the Central Nervous System, ed 4 revised. Lyon: World Health Organization, 2016

13. Li YM, Suki D, Hess K, Sawaya R: The influence of maximum safe resection of glioblastoma on survival in 1229 patients: Can we do better than gross-total resection? J Neurosurg 124:977-988, 2016

14. Lima GL de O, Duffau H: Is there a risk of seizures in "preventive" awake surgery for incidental diffuse low-grade gliomas? J Neurosurg 122:1397-1405, 2015

15. Lima GL de O, Zanello M, Mandonnet E, Taillandier L, Pallud J, Duffau H: Incidental diffuse low-grade gliomas: from early detection to preventive neuro-oncological surgery. Neurosurg Rev 39:377-384, 2016

16. Mandonnet E, De Witt Hamer P, Poisson I, Whittle I, Bernat $\mathrm{AL}, \mathrm{Bresson} \mathrm{D}$, et al: Initial experience using awake surgery for glioma: oncological, functional, and employment outcomes in a consecutive series of 25 cases. Neurosurgery 76:382-389, 2015

17. Muto J, Dezamis E, Rigaux-Viode O, Peeters S, Roux A, Zanello M, et al: Functional-based resection does not worsen quality of life in patients with a diffuse low-grade glioma involving eloquent brain regions: a prospective cohort study. World Neurosurg 113:e200-e212, 2018

18. Nossek E, Matot I, Shahar T, Barzilai O, Rapoport Y, Gonen $\mathrm{T}$, et al: Failed awake craniotomy: a retrospective analysis in 424 patients undergoing craniotomy for brain tumor. J Neurosurg 118:243-249, 2013

19. Nossek E, Matot I, Shahar T, Barzilai O, Rapoport Y, Gonen $\mathrm{T}$, et al: Intraoperative seizures during awake craniotomy: incidence and consequences: analysis of 477 patients. Neurosurgery 73:135-140, 2013

20. Oushy S, Sillau SH, Ney DE, Damek DM, Youssef AS, Lillehei KO, et al: New-onset seizure during and after brain tumor excision: a risk assessment analysis. J Neurosurg 128:17131718, 2018

21. Pallud J, Audureau E, Blonski M, Sanai N, Bauchet L, Fontaine D, et al: Epileptic seizures in diffuse low-grade gliomas in adults. Brain 137:449-462, 2014

22. Pallud J, Capelle L, Taillandier L, Fontaine D, Mandonnet E, Guillevin R, et al: Prognostic significance of imaging contrast enhancement for WHO grade II gliomas. Neuro Oncol 11:176-182, 2009

23. Pallud J, Dezamis E: Functional and oncological outcomes following awake surgical resection using intraoperative cortico-subcortical functional mapping for supratentorial gliomas located in eloquent areas. Neurochirurgie 63:208-218, 2017

24. Pallud J, Rigaux-Viode O, Corns R, Muto J, Lopez Lopez C, Mellerio C, et al: Direct electrical bipolar electrostimulation for functional cortical and subcortical cerebral mapping in awake craniotomy. Practical considerations. Neurochirurgie 63:164-174, 2017

25. Roca E, Pallud J, Guerrini F, Panciani PP, Fontanella M, Spena G: Stimulation-related intraoperative seizures during awake surgery: a review of available evidences. Neurosurg Rev 43:87-93, 2020

26. Schucht P, Ghareeb F, Duffau H: Surgery for low-grade glioma infiltrating the central cerebral region: location as a predictive factor for neurological deficit, epileptological outcome, and quality of life. J Neurosurg 119:318-323, 2013

27. Serletis D, Bernstein M: Prospective study of awake craniotomy used routinely and nonselectively for supratentorial tumors. J Neurosurg 107:1-6, 2007

28. Skardelly M, Brendle E, Noell S, Behling F, Wuttke TV, Schittenhelm J, et al: Predictors of preoperative and early postoperative seizures in patients with intra-axial primary and metastatic brain tumors: a retrospective observational single center study. Ann Neurol 78:917-928, 2015

29. Spena G, Schucht P, Seidel K, Rutten GJ, Freyschlag CF, D'Agata F, et al: Brain tumors in eloquent areas: a European multicenter survey of intraoperative mapping techniques, intraoperative seizures occurrence, and antiepileptic drug prophylaxis. Neurosurg Rev 40:287-298, 2017

30. Still MEH, Roux A, Huberfeld G, Bauchet L, Baron MH, 
Fontaine D, et al: Extent of resection and residual tumor thresholds for postoperative total seizure freedom in epileptic adult patients harboring a supratentorial diffuse low-grade glioma. Neurosurgery 85:E332-E340, 2019

31. Szelényi A, Bello L, Duffau H, Fava E, Feigl GC, Galanda $\mathrm{M}$, et al: Intraoperative electrical stimulation in awake craniotomy: methodological aspects of current practice. Neurosurg Focus 28(2):E7, 2010

32. von Elm E, Altman DG, Egger M, Pocock SJ, Gøtzsche PC, Vandenbroucke JP: The Strengthening the Reporting of Observational Studies in Epidemiology (STROBE) statement: guidelines for reporting observational studies. PLoS Med 4:e296, 2007

33. Wang YC, Lee CC, Takami H, Shen S, Chen KT, Wei KC, et al: Awake craniotomies for epileptic gliomas: intraoperative and postoperative seizure control and prognostic factors. J Neurooncol 142:577-586, 2019

34. Wieser HG, Blume WT, Fish D, Goldensohn E, Hufnagel A, King D, et al: ILAE Commission Report. Proposal for a new classification of outcome with respect to epileptic seizures following epilepsy surgery. Epilepsia 42:282-286, 2001

35. Wu AS, Trinh VT, Suki D, Graham S, Forman A, Weinberg JS, et al: A prospective randomized trial of perioperative seizure prophylaxis in patients with intraparenchymal brain tumors. J Neurosurg 118:873-883, 2013

36. Yordanova YN, Moritz-Gasser S, Duffau H: Awake surgery for WHO Grade II gliomas within "noneloquent" areas in the left dominant hemisphere: toward a "supratotal" resection. Clinical article. J Neurosurg 115:232-239, 2011
37. Zachenhofer I, Donat M, Oberndorfer S, Roessler K: Perioperative levetiracetam for prevention of seizures in supratentorial brain tumor surgery. J Neurooncol 101:101-106, 2011

\section{Disclosures}

The authors report no conflict of interest concerning the materials or methods used in this study or the findings specified in this paper.

\section{Author Contributions}

Conception and design: Pallud, Oppenheim, Varlet. Acquisition of data: Pallud, Zanello, Trancart, Parraga, Tauziede-Espariat, Sauvageon, Dezamis. Analysis and interpretation of data: Pallud, Zanello, Trancart, Parraga. Drafting the article: Pallud, Zanello. Critically revising the article: Pallud, Zanello, Roux, Zah-Bi, Edjlali, Tauziede-Espariat, Sauvageon, Sharshar, Oppenheim, Varlet, Dezamis. Reviewed submitted version of manuscript: all authors. Approved the final version of the manuscript on behalf of all authors: Pallud. Statistical analysis: Pallud. Administrative/ technical/material support: Pallud. Study supervision: Pallud.

\section{Correspondence}

Johan Pallud: Hôpital Sainte-Anne, Paris, France. j.pallud@ ghu-paris.fr. 